\title{
Review of: "Global carbon budget of reservoirs is overturned by the quantification of drawdown areas"
}

\author{
Ruihong $\mathrm{Yu}^{1}$ \\ 1 Inner Mongolia University
}

Potential competing interests: The author(s) declared that no potential competing interests exist.

This study estimated the extent of reservoir drawdown areas globally and to identify drivers influencing the extent of drawdown areas as well as their spatial and temporal patterns. And then re-assessed global estimates of reservoirs' $\mathrm{C}$ budget by partitioning total emissions into fluxes from the water surface and from the drawdown area. The study is to reduce the upscaling uncertainty of $C$ fluxes from reservoirs. The intent of the manuscript is very good, however, there are several issues which are of concern to me.

1. The drawdown area was maximum area subtraction the current monthly area, but the drawdown had a certain slope, which make the actual area larger than the subtraction between maximum area and current area. This would underestimate the area of the global drawdown area and thus increase the uncertainty of the $\mathrm{C}$ budget.

2. The GHG emissions was showed obvious temporal variations. The Upscaling of GHG emissions in this study: How to consider the area and emission rate time correspondence.

3. The emission rates used in this study are from 47 studies, which is too small in relation to the number of reservoirs studied, and did not cover southern Africa, India, and Southeast Asia in terms of geographic distribution. And the time range of cited fluxes were outside the time range of drawdown.

4. In this study, the mean annual drawdown area of reservoirs was related to latitude. And whether the cited reservoirs also have latitude distribution characteristics?

5. The definition of drawdown was different in different studies, whether it would affect the accuracy of emission rates.

6. At high latitudes, there are distinct seasons. Winter may be excluded from the measurement of emission rates in the drawdown. How to account for different time lengths when calculating the global average flux.

7. Why complete filling of reservoirs was defined by a drawdown area smaller than $5 \%$ of Areamax. For a small reservoir, $5 \%$ is insignificant, but for a large reservoir, this value cannot be ignored. 